cius de Monte Galea ${ }^{25}$ aus Prag den Schlangenaugen eine Arbeit gewidmet und ihre Heilwirkung gegen Vergiftungen angepriesen. Scheuchzer wußte mit diesen Resten besseres anzufangen; er hat der Erkenntnis zum endgültigen Durchbruch verholfen, daß die Fossilien Überreste von Tieren sind, die einst wirklich gelebt haben.

B. Peyer.

\title{
Schweizerische Bibliographie der Geschichte der Naturwissenschaften und der Medizin. I938 - 1943, III. Serie
}

\section{Neuere Paracelsus-Literatur*}

Achelis, J. D. Der Arzt Paracelsus. Die Pause, 6. Jahrgang, 9. April, Wien 1941, (illustr.).

Acta Paracelsica. Im Auftrag der Paracelsus-Gesellschaft herausgegeben von Darmstädter, Koch \& Schröter. Heft 1-5, München 1930-1932.

Albrecht, Alice Sus. Theophrastus Paracelsus. Von seiner Auffassung über Mensch und Natur. Gesundheit und Wohlfahrt, Zürich, Juli 1941.

Allendy, R. Le médecin maudit. Gallimard, Paris 1937.

- - Paracelso, il medico maledetto. Fr. Bocca, Milano 1942.

Artelt, Walter. Paracelsus im Urteil der Medinzinhistorik. Fortschr. d. Medizin 50, Nr. 22, (1932).

- - Paracelsus und seine Zeit in Zeittafeln. «Theophrastus Paracelsus» von Jaeger 1941, vide Jaeger.

Bayon, H. P. Paracelsus: personality, doctrines and his alleged influence in the reform of medicine. Proceedings of the Royal Society of Medicine, London, 35, 69-76, Section of the History of Medicien 9-16. (1941).

Bazala, Vlad. Paracelsus in Kroatien. Neue Ordnung, Zagreb, 21. Sept. 1941.

25 Ephemerides der Leopoldina, Annus IX/X der ersten Decuria, Observatio 119; \&De oculis serpentum et linguis Melitensibus».

* Da es zur Zeit unmöglich ist, die internationale Paracelsus-Literatur auch nur mit annähernder Vollständigkeit zu registrieren, wurde die wichtigere Paracelsus-Literatur bis 1930 zurück verfolgt. Für weitere bibliographische Angaben bin ich den Lesern dankbar. Bis 1930 wurde die Paracelsus-Literatur durch K. Sudhoff in «Nachweise zur ParacelsusLiteratur» (Beilage zu den Acta Paracelsica 1-5, 1930-1932) in 1089 Nummern zusammengestellt.

Eine größere Zahl mir unbekannter Literaturzitate verdanke ich der Güte von P. Dr. Ildefons Betschart in Einsiedeln, welcher in den Nova Acta Paracelsica Bd. I. (Birkhäuser Basel 1944) ebenfalls eine durch gegenseitige Ergänzung konforme Paracelsusbibliographie erscheinen läßt. 
Betschart, Ildefons. Theophrastus Paracelsus. Der Mensch an der Zeitenwende, Einsiedeln 1941, Verlag Benziger. 171 S. 2. A. 1942 Ebenda.

- Paracelsus. Schweiz. Monatshefte 1941, 347-358 (Oktoberheft).

- - Paracelsus in der Sage. Atlantis (illustr.), Sept. 1941.

- - Paracelsus-Bibliographie. Nova Acta Parac. I. Bd. 1944, Birkhäuser Basel.

Bier, D. Paracelsus und die sog. Tartarenkrankheit. Kölnische Zeitung, 21. März 1943.

Bigler, Walter. Theophrastus Paracelsus. St. Galler Tagblatt, 24. u. 25. Sept. 1941.

- Grundsätzliches zur Paracelsus-Forschung. Nova Acta Parac. I. Bd. 1944. Birkhäuser, Basel.

Bircher, Eugen. Theophrastus Paracelsus von Hohenheim aus Einsiedeln, 19431541. Große Schweizer Forscher, herausgegeb. v. Ed. Fueter, Atlantis-Verlag 1938 , p. $37-39$.

- - Theophrastus Paracelsus. Große Schweizer. Atlantis-Verlag Zürich 1938, p. $61-65$.

- Theophrastus Paracelsus von Hohenheim. Schweiz. Hochschulzeitung März 1939, S. 381-391.

Birchler, Linus. Das Erbgut des Paracelsus. Gesundheit und Wohlfahrt 1940, Heft $3-5$.

- Paracelsus in bildender Kunst und Dichtung. Neue Zürcher Nachrichten, 3. Okt. 1941.

- - Fischer, Hans und Medicus, Fritz. Beiträge zur Charakteristik von Theophrastus Paracelsus. Basel, Benno Schwabe \& Co. 1936.

Bittel, Karl. Paracelsus-Dokumentation, Referatblätter zum Leben und Werk Theophrastus von Hohenheim, Blatt A 44 «Para und Paracelsus». Herausgegeben vom Paracelsus-Museum Stuttgart (1941).

- Korrekturen zur Paracelsus-Biographie; über die Notwendigkeit eines dokumentarischen Quellenwerkes. Hippokrates, Stuttgart 1943. S. 30 ss.

- - Ist Paracelsus in der Kaigasse gestorben? Hippokrates, Stuttgart 1942. S. $129 \mathrm{ss}$.

- Ist Hohenheim 1493 oder 1494 geboren? Die medizinische Welt, Berlin 1942 , p. $\cdot 1163$.

- Zur Genealogie der Bombaste von Hohenheim. Münchner Medizin. Wochenschrift, 1942 p. 359 ss.

- - Ist der Beiname «Paracelsus» am Oberrhein entstanden? Ztschr. Gesch. d. Oberrheins, Neue Folge, 56, 668 (1943, Karlsruhe).

- Paracelsus am Oberrhein. Straßburger Monatshefte 1943, p. 226-233.

_- «Paracelsus» genannt ... Wiener Medizin. Wschr. 1943 p. 451.

- Zum 450. Geburtsjahr von Paracelsus. Hohenheim ist 1493 oder 1494 geboren. Hippokrates, p. 618 ss, 27. Okt. 1943. 
- Paracelsus: Autobiographisches. Leipzig 1943, Reclam-Bändchen 7567/68.

- Las Paracelsus auf der Universität Basel oder auf einem freien Lehr. stuhl? Die Medizinische Welt, Berlin 1943, p. 13 ss.

- - Geburt, Kindheit und Jugend des Paracelsus. Europäischer Wissenschafts-Dienst, Berlin 1943, Nr. 7.

- - Die Kindheit Theophrasts in Einsiedeln. Fragen nach dem historischen Wahrheitsgehalt der Paracelsus-Biographien. Nova Acta Parac. I. Bd. 1944, Birkhäuser, Basel.

Brinkmann, Donald. Mythos und Logos im Weltbild des Paracelsus. Nova Act a Paracelsica Bd. I. S. 109-134.

Brunn, Walter A. L. von. Ärztliche Erfahrung bei Paracelsus. Münch. med. Wschr. 1941, 1044.

- - Paracelsus und die Chirurgie. Zentralbl. f. Chirurgie, 68, (1941).

- - Paracelsus und seine Schwindsuchtslehre. Praktische Tuberkulose Bücher, 26. Heft. Leipzig: G. Thieme 1941, $62 \mathrm{~S}$.

- Zur Elementarlehre des Paracelsus. Sudhoff's Arch. Gesch. Med. 34, (1941).

Bugge, Günther. Leonhard Thurneyßer in Tirol. Z. angew. Chem. 53, 190-192 (1940).

Daday, Andras. Paracelsus Theophrastus. Egeszség, Budapest, 15. April 1942.

Damur, C. Paracelsus. Neue Schweiz. Rundschau, Oktober 1941.

Darmstaedtter, Ernst. De natura rerum. Eine kritische Studie. Janus (Arch. Int. Hist. Méd.) 37, 1-18, 48-62, 109-115 (1933).

Diepgen, Paul. Paracelsus 1493-1541. Die großen Deutschen. Neue deutsehe

Biographie: Herausgegeben von W. Andreas und W. von Scholz. Bd. 1, Berlin 1935 , S. $520-531$.

- Hippokrates oder Paracelsus. Kleine Hippokrates-Bücherei, Band 9 , Hippokrates Verlag, Stuttgart 1937. 30 S.

- Die Frauenheilkunde des Paracelsus. Hippokrates 1940, S. 130-137.

- Der Arzt Paracelsus und die moderne Medizin. Münch. med. Wschr. 1941, 1041.

- - Theophrast von Hohenheim, genannt Paracelsus, der Arzt zwischen den

Zeiten. Forschungen und Fortschritte 1941, 293-299 Nr. 27-28.

Dingelday. Theophrastus von Hohenheim, gen. Paracelsus. In "Theophrastus Paracelsus», v. Jaeger, vide Jaeger.

Englert, Ludw. Von der rechten Heilkunst, ein Paracelsus-Lesebuch. Hippokrates-Verlag, Stuttgart 1939.

- - Paracelsus, Mensch und Arzt. Limpert, V., Berlin 1941.

Fischer, Hans. Helleborus im Altertum und bei Paracelsus. Schweiz. med. Wschr. 66, 484, (1936). 
- - Der junge Paracelsus. Verh. schweiz. natf. Ges. 1941, 209-211 (Referat) Schweiz. med. Wschr. 71, 1145, (1941).

- - Die kosmologische Anthropologie des Paracelsus als Grundlage seiner Medizin. Ein Beitrag zum Verständnis des Arztes Paracelsus. Verh. d. naturf. Ges. in Basel, Bd. 69, 51 S. (1941).

- - siehe auch: Birchler, L. ...

Fritsche, Herb. Der erste Mensch der Neuzeit. Berliner Lokalanzeiger, Berlin 21. Sept. 1941.

Führer durch die Paracelsus-Gedächtnis-Ausstellung. Hippokrates-Verlag Marquardt \& Co., Stuttgart 1941. 91 S.

Gabathuler, Matthäus. Vadian und Paracelsus. St. Galler Tagblatt, Nr. 526, (1941).

Ganzenmüller, Wilhelm. Paracelsus und die Alchemie des Mittelalters. Z. angew. Chem. 54, 427-431 (1941).

Geilinger, Max. Das Spiel von Paracelsus. Rascher \& Co., Zürich 1941.

Greil, Alfred. Die paracelsische Renaissance. Schweiz. med. Wschr. 71, 1134, (1941).

- - Paracelsus redivivus. (Zum 450. Geburtstage.) Nova Acta Parac. I. Bd. S. 159-168, 1944, Birkhäuser, Basel.

Gruber, B. Theophrastus Paracelsus. Niederländische Tageszeitung, Hannover, 21. Sept. 1941.

Gyr, Martin. Zur Frage der Geburtsstätte. Safrascht: Beilage der «Neuen Einsiedler Zeitung» Oktober 1941, Nr. 66.

- - Vom Wappen Paracelsus'. Beilage der "Neuen Einsiedler Zeitung» (Nu einischt Safrascht) Oktober 1941, Nr. 66.

Haemmerli-Schindler, Theodor. Paracelsus. Zum 400jährigen Todestage. Praxis, Schweiz. Rundschan f. Med. 30, 640-643 (1941).

Fartlaub, G. F. Paracelsus und die Künstler. Frankfurter Zeitung, 25. Sept. 1941.

Hartmann, Hans. Paracelsus - eine deutsche Vision. Neues Volk. Berlin. Wien 1941.

Heimsoeth, H. Paracelsus als Philosoph. Disch. Vierteljschr. f. Literaturwissenschaft u. Geistesgesch. Jahrg. 19 (1941).

Heinrichs, Heinr. Paracelsus und die Bergkrankheiten. Deutsche BergwerksZeitung, Düsseldorf, 24. Sept. 1941.

Henggeler, Rudolf. Paracelsus: Bild des damaligen Einsiedeln - Wilhelm Bombast von Hohenheim. Zürichsee-Zeitung, Stäfa, 24. Sept. 1943.

Hiller, Joh. Erich. Paracelsus und de Boodt als Vorläufer neuzeitlicher Mineralogie. Naturwiss. 30, 563-565 (1942).

Hooykaas, Reijer. Die chemische Verbindung bei Paracelsus. Sudhoff's Arch. Gesch. Med. 32, 166-175 (1939). 
- Paracelsus' Bedeutung für die Chemie. Chem. Weekbl. 38, 527-529 (1941).

Jaccard, R. Hommage à René Allendy. Nova Acta Parac. I. Bd. 1944, Birkhäuser, Basel.

Jacobi, Jolan. Paracelsus. Lebendiges Erbe. Auslese aus den Werken. Buchh. Rascher \& Co., Zürich 1942.

Jaeckle, E. Paracelsus. Seine Weltschau in Worten seines Werkes. AtlantisVerlag Zürich (1942).

Jaeger, Fritz. Theophrastus Paracelsus 1493-1541, Bilderbuch mit verschiedenen Aufsätzen, Mora Salzburg 1941.

Jenny, Ed. Paracelsus und die Grundlagen der ärztlichen Kunst. Schweiz. med. Wschr. 71, 1125 (1941).

- Paracelsus, der Arzt. Nova Acta Parac. I. Bd. S. 69-81, Birkhäuser, Basel, 1944.

Jung, C. G. Paracelsus als Arzt. Verh. schweiz. natf. Ges. 1941, 207-209 (Referat). Schweiz. med. Wschr. 71, 1153 (1941).

- - Paracelsica. Paracelsus als Arzt und Paracelsus als geistige Erscheinung. Zwei Vorlesungen über den Arzt und Philosophen Theophrastus. Zürich u. Leipzig: Rascher Verlag 1942. 188 S. ill.

- - Paracelse, le Précurseur. Formes et couleur, Held, Lausanne, 2-3, 1941.

- Psychologie und Alchemie (mit 270 Illustrationen). Verlag Rascher Zürich 1944. $696 \mathrm{~S}$.

Kaiser, H. Theophrastus Paracelsus. Die Krankenhaus-Apotheke. Sept. 1941. Karcher, Hans. Paracelsus, Stadtarzt von Basel. Schweiz. med. Wschr. 71, 113 (1941).

- Paracelsus, Stadtarzt von Basel. Nova Acta Paracelsica I. Bd. S. 83 bis 102, Birkhäuser, Basel 1944.

Kayser, H. Das Formendenken des Paracelsus. Nova Acta Paracelsica Bd. I. S. 103-108, Birkhäuser Basel 1944.

Kellner, A. W. Paracelsus. Die Umschau, Frankfurt a. M., 28. Sept. 1941.

Kolbenheyer, E. G. Paracelsus, der Deutsche. Hamburger Fremdenblatt, 21. Sept. 1941.

- - Paracelsus. Romantrilogie, ungekürzte Volksausgabe. 1941. LangenMüller, München.

Leibbrand, Werner. Paracelsus. Frankfurter Zeitung, 24. Sept. 1941.

- - Verhältnis zu Paracelsus. Atlantis. Sept. 1941 (illustr.).

Lejeune, Fritz. Theophrastus von Hohenheim. Paracelsus. (Mit Abb. u. 2 Kartenskizzen.) Verlag H. Hillger, Berlin 1941, $60 \mathrm{~S}$.

- War Paracelsus seiner Zeit voraus? Natur und Kultur, Wetzel, München, 40. Jahrg. 1.-2. Heft (1943). 
Lienhardt, B. Wo lag das Geburtshaus des Paracelsus? Einsiedler Anzeiger, 3. Okt. 1941 .

- Wer war seine Mutter? Einsiedler Anzeiger, 3. Okt. 1941.

- - und R. Henggeler. Das Paracelsus-Haus am Etzel und seine Bewohner. Neue Zürcher Nachrichten. 3. Okt. 1941.

- - und Schönenberger, K. Medizingeschichtliches aus Einsiedeln. - Paracelsus. Illustr. Sonderdruck aus «Einsiedler Anzeiger», 1941.

Lindroth, Sten. Hiàrne, Block och Paracelsus. En redogörelse för Paracelsusstriden 1708-1709. Lychnos 1941 S. 229-247. Uppsala 1941 (Schwedisch).

-_ - Paracelsismen i Sverige till 1600 talets-mtt. (Schwedisch.) Lychnos Bibliothek 7, 540 S. Uppsala 1943. Almquist u. Wiksells.

Lopez, I. Ibor. Paracelso. Arriba, Madrid, 24. Sept. 1941.

Medicus, Fritz. Die wissenschaftliche Bedeutung des Paracelsus. Schweiz. med. Wschr. 1936, S. 17-20.

- Paracelsus in der philosophischen Bewegung seiner und unserer Zeit. Nova Acta Parac., Bd. 1, Birkhäuser Basel 1944, S. 45-68.

- - siehe auch: Birchler, L...

Milt, Bernhard. Conrad Geßner und Paracelsus. Schweiz. med, Wschr. 59, Nr. 18-19 (1929).

- Paracelsus und Zürich. Vierteljschr. natf. Ges. Zürich 86, 321 (1941).

- Theophrastus Paracelsus. Der Freisinnige, Wetzikon, 22. Sept. 1941.

Murphy, K. Marvellous Paracelsus. Sixth Series, Nr. 192 (1941).

Neergard, K. von. Was lehrt uns Paracelsus für die weitere Entwicklung der Medizin? Hippokrates 1942, 123-129.

Nenninger, J.E. Zur Entwicklung des Arzneischatzes im Zeitalter des Paracelsus. Ciba Zeitschr. Sonder-Nummer 18. Juni 1942: Die Arznei und ihre Zubereitung. Basel 1942, 44-51.

Netzhammer, Raymund, Erzbischof. Paracelsus zwischen zwei Jahrhundertfeiern. Schweiz. med. Wschr. 71, 1109 (1941).

- Zur Würdigung des Paracelsus in Einsiedeln. Meinradsraben, 1941.

Nova Acta Paracelsica. I. Jahrbuch der Schweiz. Paracelsusgesellschaft. Birkhäuser Basel 1944. 192 S. (illustr.).

Oesterle, Friedrich. Die Anthropologie des Paracelsus. Neue Deutsche Forschung, Abt. Charakterologie usw. Bd. 5, Berlin 1937, Junker und Dünnhaupt.

Oettli, Th. Paracelsus und St. Moritz. Schweiz. med. Wschr. 71, 1121 (1941).

Paracelsus. Vom Holz Guajaco gründlicher Heilung. 1529. Neu bei: O. Barth, München 1926 (bei Sudhoff nicht aufgeführt).

Partington, J. R. Paracelsus (1493-1541). Nature 148, 332-34 (1941).

Peternell, P. Der König der Ärzte. Ein Paracelsus-Roman. Berglandbuch, Salzburg 1941. 
Peuckert, Will-Erich. Pansophie, ein Versuch zur Geschichte der weißen und schwarzen Magie. Stuttgart 1936.

- - Begnadet zum Suchen. Die Pause, 6. Jahrg. 9. Heft, Wien 1941 (illustr.).

- - Paracelsus. Die Geheimnisse. Ein Lesebuch aus seinen Schriften. Dieterichsche Buchhandlung. Leipzig (illustriert), Bd. 83, 1941.

- Theophrastus Paracelsus. Stuttgart und Berlin: W. Kohlhammers Verlag 1941, 472 S. 10 pl. - 2. verb. A. Stuttgart-Berlin 1943.

Reclam, E. H. Die Gestalt des Paracelsus in der Dichtung. Studien zu Kolbenheyers Trilogie. Reclam, Leipzig 1938.

Roemisch, Bruno. Theophrastus Paracelsus und der Norden. Deutsche Medizin. Wschr. 19. Sept. 1941.

Römer, Hermann. Die Sippe des Paracelsus, Abstammung und Schicksal der Bombaste von Hohenheim. Stuttgarter Neues Tagblatt, Stuttgart, 24. Aug. 1941.

Rosen, George, siehe: Temkin, C. L... .

Salzer, E. C. Theophrastus Bombast von Hohenheim, detto il Paracelso. Rinasciata, Florenz 1940.

Schaefer, Walter. Der Ketzer von Basel. Der Führer, Karlsruhe, 21. Sept. 1941.

Schär, R. Paracelsus. Zum 400. Todestag des großen Mediziners der Renaissance am 24. September 1941. Schweiz. Apoth.-Ztg. 79, 565-567 (1941).

Schmaltz, Dieter. Pflanzliche Arzneimittel bei Theophrastus von Hohenheim, genannt Paracelsus. Hippokrates-Verlag, Marquardt \& Co., Stuttgart 1941. $87 \mathrm{~S}$.

Schönenberger, Karl. Theophrastus Paracelsus. - Der Reformator der Heilkunde. Einsiedler Anzeiger, 3. Okt. 1941, in «Alte und Neue Welt», 1941, S. 541 ss. 50 (illustr.).

Schreyer, Lothar. Der gefangene Glanz. Aus den Werken des Paracelsus. Freiburg i. Br., Caritas, 1940.

Schroeter, Joachim. Chemisches und Mineralogisches bei Paracelsus. Neue Zürcher Ztg. 1941, Nr. 1982 (7. Dez.) und Nr. 1994 (8. Dez.).

- - Die Stellung des Paracelsus in der Mineralogie des 16. Jahrhunderts. Schweiz. Min. Petr. Mitt. 21, 313-331 (1941).

Schuhmacher, Jos. Das Übergeschichtliche bei Paracelsus. Nationalzeitung, Essen, 15. Juli 1943.

Schwarber, K. Die Paracelsus-Ausstellung der Basler Universitätsbibliothek. Schweizer Sammler, 9/10, 1941.

Senatra, Edoardo. Teofrasto Paracelso. Il Messagero, Rom, 25. Sept. 1941.

Sievers, Gerda. Die Naturanschauung des Paracelsus. Würzburg 1937.

Sigerist, Henry, E. Laudanum in the works of Paracelsus. Bull. of the History of Medicine 9, 530-544 (1941). 
- Paracelsus in the light of four hundred years. The March of Medicine,

28-51, New York, Columbia University Press (1941).

- - siehe auch: Temkin, C. L. ...

Silber, Max. Das Bildnis Wilhelms von Hohenheim. Neue Einsiedler Zeitung, Einsiedeln, 27. Nov. 1942.

- - Paracelsus und Salzburg. In Jaeger «Theophrastus Paracelsus» sowie:

Die Pause, 6. Jahrg. 9. Heft (illustr.), Wien 1941.

Sills-Fuchs, Martha. Paracelsus und wir. Studie über die Persönlichkeit des

Theophrastus von Hohenheim. Müller, Planegg v. München 1941 (illustr.).

Spühler, V. Paracelsus. Schweiz. Archiv f. Tierheilkunde, Sept. 1941.

Spunda, Franz. Paracelsus. Ein Mann sucht die Wurzel aller Dinge und des

Geistes. Chemnitzer Tageblatt, 14. Sept. 1941.

- Das Weltbild des Paracelsus. Verlag Wilh. Andermann, Wien 1942.

Stadlinger, Herm. Paracelsus. Chemiker-Zeitung, Köthen. 17. Sept. 1941.

Sticker, Georg. Paracelsus, ein Lebensbild. Nova Acta Leopoldina N. F. 10,

Nr. 66, S. 31-126 (1941).

- - Ein Gespräch des Königs Ferdinand mit Paracelsus. Nova Acta Leopoldina N. F. 10, Nr. 69, S. 267-278 (1941).

Strebel, Joseph. Über die drei Grundgesetze der paracelsischen Schriften. Das

Gesetz der Periodizität und das Großgesetz der sphärisch-astralen Entsprechungen, Korrespondenzen, oder Korrelationen. Schweiz. med. Wschr. 71, 1127 (1941).

- - Quid est Archeus? Archeus als individuelles und kollektives Seelenprinzip des Mikro- und Makrokosmos. Schweiz. med. Wschr. 71, 1130 (1941).

- - Paracelsus als Erbforscher, Eugeniker und Praktiker der Vitaminlehre.

Schweiz. med. Wschr. 71, 1133 (1941).

- Kausalität und Finalität im Paracelsischen Sinne. Schweiz. med. Wschr.

71, 1101, (1941).

- Der Schalk in Paracelsus. Schweiz. med. Wschr. 71, 1137, (1941).

- - Theophrastus von Hohenheim, genannt Paracelsus. Zur 4. Jahrhundert-

feier seines Todes, 24. September 1541-1941. Der Stein der Weisen. Luzera,

E. Haag, 1941, $79 \mathrm{~S}$.

- - Paracelsus und die Augenheilkunde. Klin. Monatschr. f. Augenheilkunde. 107, 528-533 (1941).

- Lob des Paracelsus. Neue Zürcher Zeitung, 20. April 1941.

- Paracelsus-Studien I. und II. Lob des Paracelsus (erstmals in der Neuen Zürcher-Zeitung, Nr. 609, 1941). Die Geistgestalt des Theophrastus von

Hohenheim. Weltenschau des Paracelsus (1941 in Ragaz uraufgeführt). Herausgegeben von der Schweiz. Bibliophil. Gesellschaft.

- - Paracelsus, der Schalk. Luzerner Neueste Nachrichten, 21. Sept. 1941,

- Vererbungsstudien an Paracelsus. Schweiz. med. Wschr. 1943, 1582. 
- Paracelsus. Seine Weltschau in Worten des Werkes, Atlantisverlag Zürich 1942. Nova Acta Parac. I. Bd. S. 155-158, 1944, Birkhäuser, Basel. - - Nachweise von Erbeinflüssen bei Paracelsus mit Rekonstruktion des Erscheinungsbildes der Einsiedler Mutter des Hohenheimers. Gedruckt, aber noch nicht veröffentlicht.

- Stand und Aufgaben der schweizerischen Paracelsusforschung. Nova Acta Paracelsia Bd. I. S. 28-36, Birkhäuser Basel 1944.

- Dr. Hans Locher über Paracelsus anno 1851. Nova Acta Paracelsica Bd. I. S. 135-154, Birkhäuser, Basel 1944.

Strunz, Franz. Zur Lebensgeschichte des Theophrast von Hohenheim. Blätter für deutsche Philosophie 6, 338, (1932).

- Theophrastus Paracelsus. Idee und Problem seiner Weltanschauung. Salzburg-Leipzig 1937, 2 A.

- Paracelsus als Naturforscher. Chem. Ztg. 65, 421-425 (1941).

-- - Theophrastus von Hohenheim, genannt Paracelsus. Die Pause, 6. Jahrg., 9. Heft (illustr.), Wien 1941.

Sudhoff, Karl. Paracelsus. Ein deutsches Lebensbild aus den Tagen der Renaissance. Meyers kleine Handbücher, Nr. 1, Bibliographisches Institut A. G., Leipzig (1936). $156 \mathrm{~S}$.

Temkin, C. Lilian, George Rosen, Gregory Zilboorg, Henry E. Sigerist. Four treatises of Theophrastus von Hohenheim called Paracelsus, translated from the original German, with introductory essays. Edited, with preface by Henry E. Sigerist. XII +256 p. Publications of the Inst. of the Hist. of Med., The Johns Hopkins Univ.; Second Series: Texts and documents; vol. I. Baltimore 1941.

Testi, Karl. Paracelso e l'interpretazione chimico-fisica della vita. Collana di Storia della chimica. Nr. 3, Mediterranea, Rom 1942.

Veil, Wolfgang H. Paracelsus, zum 400. Todestag. G. Fischer, Jena 1942.

Vischer, A. L. Paracelsus in Basel. Praxis, Schweiz. Rundschau f. Med. 30, $644-647$ (1941).

Vogt, Alfr. Medizin und Naturphilosophie bei Theophrastus Paracelsus. Deutsche med. Wschr. 19. Sept. 1941.

Walden, Paul. Paracelsus (1493-1541) und seine Bedeutung für die Chemie. (Vortrag.) Forschungen und Fortschritte 16, 210-212, (1940).

- Paracelsus und seine Bedeutung für die Chemie. Angewandte Chemie 54, 421-427 (1941).

- - Paracelsus als Chemiker. Chemiker-Zeitung, Köthen, 65, 351 (1941).

- Paracelsus. - Zaubermeister und Wohltäter. Velhagen \& Klasings Monatshefte, Berlin Sept. 1941.

Waltari, Mika. Paracelsus Baselissa (Paracelsus in Basel). Historisches Schauspiel in 5 Akten. Porvoo/Helsinki 1943. 
Waltershausen, Bodo Sartorius Freiherr von. Paracelsus am Eingang der deutschen Bildungsgeschichte. Forschungen z. Gesch. d. Philosophie und der Pädagogik, Heft 16, Verlag F. Meiner, Leipzig 1936, 216 S.

- - Theophrastus Paracelsus. Entdecker der Chemie. Die Pause, 6. Jahrgang, 9. Heft, Wien 1941.

Wegener, Ilse. Paracelsus. Die Frau, Berlin, Sept. 1941.

Werle, Fritz. Paracelsus. Nova Acta Paracelsia Bd. I. S. 169-181, Birkhäuser Basel 1944.

Zeller, Eberhard. Paracelsus der Beginner eines deutschen Arzttums. Werkstätten der Stadt Halle Burg Giebichenstein (o. J. 1935?)

Zilboorg, Gregory, siehe Temkin, C. L.

Zimmermann, Walter. Der Grund der Arznei ist die Liebe. Paracelsus und die deutsche Arzneimittellehre (illustr.). Die Pause. 6. Jahrg., 9. Heft, Wien 1941.

H. Fischer,

\section{Buchbesprechungen}

Vesaliana. A Bio-Bibliography of Andreas Vesalius.. By Harvey Cushing. (Publication No 6 Historical Library, Yale University Library) Pp XXXXVIII $111+230+86$ plates. New York Schumanns 1943. 15. $-\$$.

Harvey Cushing, der klassische Meister der Neurochirurgie und einer der großzügigsten und tief interessierten Sammler medizinhistorischer Werke hat sich während 40 Jahren seines reichen Lebens mit Vesal beschäftigt. Zwanzig Jahre wandte er daran, um die für europäische Verhältnisse fast unerschwinglich gewordenen Originalien zu seiner Vesal-Bio-Bibliographie zu sammeln, welche ihn bis in die letzten Tage seines Lebens beschäftigte. Leider blieb das kostbare, mit 86 Tafeln geschmückte Werk, welches in neun Kapiteln die Hauptstationen des vesalianischen Lebenswerkes behandelt und in einem letzten, 10. Kapitel die sehr wertvolle Vesal-Bibliographie enthäl, Torso. J. F. Foulton hat das Werk seines Freundes zu Ende geführt und damit der Vesal-Forschung einen großen Dienst geleistet. - Eine ausführliche Besprechung des "Vesaliana» aus der Feder J. N. L. Bakers findet sich in Nature 153, 694, (1944).

Es darf in diesem Zusammenhang erwähnt werden, daß Harvey Cushings glänzend ausgestattete medizinhistorische Bibliothek, welche Vesal'sche Originalausgaben und die Vertreter der Renaissancemedizin in unerreichter Vollständigkeit beherbergt, seit Jahresfrist in einem Bibliotheksneubau der Yale University (als Legat Cushings) Aufstellung fand. Dorthin wird auch die 